\title{
An index of carcenogenesis using pairwise consistency
}

\section{Burzin Bhavnagri}

\author{
Email: bhavnagri@alumni.adelaide.edu.au
}

\begin{abstract}
An index is developed for elements and molecules by applying pairwise consistency to relativistic quantum mechanics. This index can be applied to study carcinogenic substances identified in studies on humans or primates. This index is found to be significantly different between carcinogenic and non-carcinogenic chemicals tested on rhesus monkeys.

An existing theory of how some chemicals cause damage to living cells is that free radicals can react with other molecules to cause cell damage or DNA mutation. A free radical is any atom or molecule that contains unpaired electrons. Historically, this meant its spin was not zero.

In quantum mechanics each molecule under consideration can be thought of as a wave function from a Hilbert space. The Hilbert space of the wave function with odd half spin is shown not to be pairwise consistent. We have previously made the hypothesis that (the spaces of wave functions of) spin zero atoms or molecules are pairwise consistent.

Now the lens space $L(3, q)$ is also pairwise consistent if $q$ is relatively prime to 3 . Thus we reason that the latter may be a limit or generalization of the Hilbert space (of wave functions of the molecule under consideration). We have defined the above index based on a function that is sensitive to both spin parity and whether it is relatively prime to 3 , the space dimensions.

The index can be used to extend the theory of how chemicals may cause damage to living cells. Based on the value of the index calculated from a molecule, that molecule can react with other molecules to cause cell damage or DNA mutation.
\end{abstract}

Keywords: Carcinogen, pairwise consistency, representational consistency, quantum mechanics 
B. Bhavnagri, An index of carcenogenesis using pairwise consistency

\section{INTRODUCTION}

Pairwise consistency has been defined in a general sense in Bhavnagri (2011). Here we specialise it to a space of wavefunctions of an atom or molecule $S$, subject to the Dirac equation. We take a quantum symmetry to be a monomorphism. Then pairwise consistency means the operator

$$
\cup\left(R_{1}, R_{2}\right)=\left\{\left(s,\left\{R_{1}(s)\right\} \cup\left\{R_{2}(s)\right\}\right) \mid s \in S\right\}
$$

is one to one for all quantum symmetries from $S$. This operator can be thought of as superimposing two wavefunctions. Pairwise consistency can be thought of as imposing a requirement that the superimposed mapping is not too different from the original wavefunction.

A key point about pairwise consistency is that we can vary the notion of structure, and in so doing obtain different results. Think of it as analogous to the way that quantum systems have different quantum numbers for shells, angular momentum and spin. Given that quantum systems are in a superposition of many states, the results we obtain can be thought of as saying if the original atom or molecule is pairwise consistent, then the superposed system is not too different from the original atom or molecule.

The Heisenberg group $H_{1}$ is the symmetry group of the one dimensional (quantum) harmonic oscillator. The Heisenberg group $H_{1}$ consists of matrices of the form below where $a, b, c \in \mathbb{R}$.

$$
\left[\begin{array}{lll}
1 & a & c \\
0 & 1 & b \\
0 & 0 & 1
\end{array}\right]
$$

Given time independent Hamiltonian defined by

$$
\begin{aligned}
H & =\frac{p^{2}}{2 m}+\frac{1}{2} m \omega^{2} x^{2} \\
p & =-i \hbar \frac{\partial}{\partial x}
\end{aligned}
$$

the wave functions $\psi$ are the solutions to the time independent differential equation

$$
H \psi=E \psi
$$

for scalar $E$. The physical meaning of these variables is the following. Position is denoted by operator $x$, momentum operator $p$, mass $m$, frequency $\omega$ and energy $E$. The quantum harmonic oscillator describes molecular orbitals.

The harmonic oscillator has solutions

$$
\begin{aligned}
\psi_{n}(x) & =\frac{1}{\sqrt{2^{n} n !}}\left(\frac{m \omega}{\pi \hbar}\right)^{1 / 4} \exp \left(-\frac{m \omega x^{2}}{2 \hbar}\right) h_{n}\left(x \sqrt{\frac{m \omega}{\hbar}}\right) \\
E_{n} & =\hbar \omega\left(n+\frac{1}{2}\right)
\end{aligned}
$$

where the polynomial $h_{n}(z)$ is called the $n$-th degree Hermite polynomial. It satisfies the recursion

$$
\begin{aligned}
h_{n}(z) & =2 z h_{n-1}(z)-2(n-1) h_{n-2}(z) \\
h_{0}(z) & =1 \\
h_{1}(z) & =2 z \\
h_{2}(z) & =4 z^{2}-2 \\
h_{3}(z) & =8 z^{3}-12 z
\end{aligned}
$$

It also has angular or rotational solutions. These solutions are derived by taking a Fourier transform. Using units where the mass and frequency of the oscillator are one, the Hamiltonian simplifies to $H=\frac{1}{2}\left(x^{2}+p^{2}\right)$. 
A Lie algebrag is a vector space together with a skew-symmetric bilinear map satisfying the Jacobi identity. For any $X, Y \in \mathfrak{g}$ the skew-symmetric bilinear map is denoted $[X, Y] \in \mathfrak{g}$. The Jacobi identity is

$$
[[X, Y], Z]+[[Y, Z], X]+[[Z, X], Y]=0
$$

for any $X, Y, Z \in \mathfrak{g}$.

The Lie algebra of $H_{1}$ consists of matrices of the form below where $x, y, z \in \mathbb{R}$.

$$
\left[\begin{array}{lll}
0 & x & z \\
0 & 0 & y \\
0 & 0 & 0
\end{array}\right]
$$

A basis for the Lie Algebra is

$$
X=\left[\begin{array}{lll}
0 & 1 & 0 \\
0 & 0 & 0 \\
0 & 0 & 0
\end{array}\right], Y=\left[\begin{array}{lll}
0 & 0 & 0 \\
0 & 0 & 1 \\
0 & 0 & 0
\end{array}\right], Z=\left[\begin{array}{lll}
0 & 0 & 1 \\
0 & 0 & 0 \\
0 & 0 & 0
\end{array}\right]
$$

The only nonzero brackets are

$$
[X, Y]=[-Y, X]=Z
$$

The Lie algebra of $H_{1}$ has the infinite dimensional representations $X: f \mapsto \pi i x \cdot f, Y: f \mapsto \frac{d f}{d x}, Z=[X, Y]$ and $Z: f \mapsto-\pi i x$ where $x \mapsto x$ is the identity map. Exponentiating the operators we obtain representations of $H_{1} e^{t X}: f \mapsto(\cos t x+i \cdot \sin t x) \cdot f, e^{t Y}: f \mapsto F_{t}(x)=f(t+x)$ and $e^{t Z}: f \mapsto e^{-\pi i t} \cdot f$. These are the rotation (acting by conjugation), translation (acting by conjugation), and time decay symmetries of the harmonic oscillator. In the Lie algebra $[X, Z]=0$ and $[Y, Z]=0$ so $Z$ corresponds to $\left[\begin{array}{lll}1 & 0 & z \\ 0 & 1 & 0 \\ 0 & 0 & 1\end{array}\right]$.

In higher dimensions $H_{n}$ is the group of $(n+2) \times(n+2)$ square matrices

$$
\left[\begin{array}{ccc}
1 & \mathbf{a} & c \\
0 & I_{n} & \mathbf{b} \\
0 & 0 & 1
\end{array}\right]
$$

where $\mathbf{a}$ is a row vector of length $n$ and $\mathbf{b}$ is a column vector of length $n$, and $I_{n}$ is the $n \times n$ identity matrix. The Lie algebra of $H_{n}$ consists of the matrices

$$
\left[\begin{array}{ccc}
0 & \mathbf{a} & c \\
0 & 0_{n} & \mathbf{b} \\
0 & \cdots & 0
\end{array}\right]
$$

where $0_{n}$ denotes the $n \times n$ zero matrix. The group $H_{3}$ is the symmetry group of the 3 -dimensional harmonic oscillator.

Given a Lie algebra a series of Lie algebras can be defined by taking brackets of the algebra with themselves. The derived series is defined by

$$
\begin{aligned}
& \mathcal{D}^{1} \mathfrak{g}=[\mathfrak{g}, \mathfrak{g}] \\
& \mathcal{D}^{k} \mathfrak{g}=\left[\mathcal{D}^{k-1} \mathfrak{g}, \mathcal{D}^{k-1} \mathfrak{g}\right]
\end{aligned}
$$

A special case of Lie algebras is when the derived series terminates after a finite number of steps, all others in the series being zero. A Lie algebra is called solvable if $\mathcal{D}^{k} \mathfrak{g}=0$ for some $k$.

The standard example of a solvable Lie algebra is the space of upper triangular matrices. The following Lemma is important because it gives us a means of identifying groups with no elements of finite order, subject to caveats. The caveat is that we are using analytic concepts that depend on the continuum hypothesis, and therefore are not in a purely algebraic setting. Also if the Lie group is defined over a base field such as $\mathbb{C}$, the base field may still contain elements of finite order. 


\section{Lemma 1. The Heisenberg group has no elements of finite order.}

Proof. $H_{n}$ is simply connected, and its Lie algebra is solvable. According to Lemma 48 in Bhavnagri (2012), if $G$ is a simply connected Lie group, and its Lie algebra is solvable, then $G$ cannot contain any non-trivial compact subgroups. In particular it contains no elements of finite order. Thus the Heisenberg group has no elements of finite order.

Referring to theorem 4 in Bhavnagri (2011), we see that the quantum harmonic oscillator will be pairwise consistent if the Heisenberg group acts freely. Since the Heisenberg group acts by conjugation, it acts freely if it has trivial center. The group $H_{1}$ has center

$$
Z\left(H_{1}\right)=\left\{\left[\begin{array}{lll}
1 & 0 & c \\
0 & 1 & 0 \\
0 & 0 & 1
\end{array}\right], c \in \mathbb{R}\right\}
$$

which we have already noted correspond to the time decay symmetries. Although we can split a molecule in two parts and split $H_{1}$ into two semi-groups we will not pursue that here. We are not in a position to apply theorem 4, but we have strong grounds to suspect all matter and light is pairwise consistent in a weak sense. We would like to develop the strong form to differentiate between elements or molecules.

This is as far as we can proceed with quantum mechanics alone, we also need the relativistic concept of spin. The reason for this is that larger symmetry groups like those of Newtonian mechanics or the unitary group do have lots of involutions. On the other hand if we are restricted to symmetries that remain in the same relatavistic frame, that is tractable from the viewpoint of pairwise consistency.

\section{DERIVATION OF THE INDEX}

\subsection{Symmetric space}

The isotropy group of a group $G$ acting on a set $M$ at $p \in M$ is the set of $g \in G$ fixing $p$ ie $I s o_{p}=\{g p=p\}$. We say that a Riemannian manifold $(M, g)$ is a symmetric space if for each $p \in M$ the isotropy group Iso $o_{p}$ contains an isometry $I_{p}$ such that $D I_{p}: T_{p} M \rightarrow T_{p} M$ is the antipodal map $-I$. In any symmetric space there is an isometry $\sigma_{p}: M \rightarrow M$ with $\sigma_{p}(p)=p$ and $D \sigma_{p}(p)=-\mathrm{id}$, and $I_{p}=D \sigma_{p}$, and this isometry is called an involution. Therefore for any space to be pairwise consistent with respect to isometries as monomorphisms, it cannot be a symmetric space. A classic example of a space which is not symmetric will follow.

According to Carey Carey et al. (1998) there are two examples of locally homogeneous spaces that are not locally symmetric (see proof of Corollary 10).

\subsection{Odd half spins and free radicals}

The Klein-Gordon equation governs elementary particles in a scalar field with spin zero, but its solutions are plane waves. This does not match the Hermite polynomial solutions of the harmonic oscillator. In chemistry many atoms have spin zero. Pairs of non-interacting electrons can have spin zero. These are tensor products of a spin $+\frac{1}{2}$ electron and spin $-\frac{1}{2}$ electron.

The Dirac equation is a Lorentz invariant wave equation $\not D \psi=\lambda \psi$. The complex valued wavefunction is replaced with a 4 tuple called a spinor subject to

$$
\not D=\sum_{\mu=0}^{3} \gamma_{u} \frac{\partial}{\partial x_{\mu}}
$$

where $\gamma_{0}, \ldots, \gamma_{3}$ were $4 \times 4$ matrices. The matrices must satisfy the Clifford relations

$$
\gamma_{\nu} \gamma_{\mu}+\gamma_{\mu} \gamma_{\nu}= \pm 2 \delta_{\nu \mu}
$$

This is the Clifford algebra $\mathrm{Cl}_{1,3}(\mathbb{C})$, so we would like to apply part (iv) of Lemma 6 in Bhavnagri (2011). If a ring has no non-trivial idempotents, it satisfies the reality property, and its automorphisms are all inner, then it satisfies the fourth equation of pairwise consistency.

We are still left with the problem of the reality condition. We can employ the Majorana map as in Zimba and Penrose Zimba and Penrose (1993), Penrose (1994) to the Riemann sphere. An equivalence of antipodal 
points and orthogonal spin states means we can replace involution with Hilbert space orthogonal in the case of $n$ odd $\left(\frac{1}{2}, \frac{3}{2}\right.$ etc). This includes the electron and proton, and non-interacting combinations of them, whose spins are given by the spin addition rule. Spins do not add as numbers, for example two spin $\frac{1}{2}$ particles may have total spin 0 or 1 .

From the above we can conclude the following theorem, but an alternative proof is also provided. The alternate proof uses the grade involution instead of antipodal points.

Theorem 1. For a spin Hilbert space whose automorphism group acts freely and never maps a spin state to its orthogonal spin state its representations are pairwise consistent.

Proof. From lemma 164 in Bhavnagri (2012) the Clifford algebra gives rise to an inner product with respect to which the grade involution is an orthogonal mapping. From theorem 67 in Bhavnagri (2012) there is an inner product with respect to which $\langle\hat{u}, u\rangle=\langle u \cdot b, u\rangle=0$. So there is an inner product with respect to which the grade involution is an orthogonal mapping. Now in our existence theorem 4 Bhavnagri (2011) we substitute preservation of orthogonality by the inner product.

The theorem above allows us to deduce the electron cannot be pairwise consistent, because the exchange antisymmetry maps $+\frac{1}{2}$ spin to $-\frac{1}{2}$ spin and these are orthogonal. Likewise tensor products of electrons with spin $\frac{1}{2} n$ and odd $n$ cannot be pairwise consistent. So free radicals cannot be pairwise consistent.

By elimination and considering the weak pairwise consistency of the harmonic oscillator we form the hypothesis that spin zero atoms are pairwise consistent.

\subsection{Lens space}

We define the lens space and show that with respect to the structure of isometry, certain lens spaces are pairwise consistent. Let $p, q \in \mathbb{N}$ be relatively prime with $1 \leq p<q$. Let $\mathbb{Z}_{q}$ be the cyclic group of order $q$. The lens space is defined by

$$
L(p, q):=S^{p} / \mathbb{Z}_{q}
$$

and it is a manifold, for details see chapter 11 of Bhavnagri (2012).

Theorem 2. The lens spaces $L(3, q)$ with $q>2$ but relatively prime to 3 are pairwise consistent, with respect to the structure of isometries.

Proof. From $k$ pointwise linearly independent vector fields, we can obtain a free action $H$ if $k \geq 1$. Simply use the vector to displace each point, in order to get an action. From theorem 70 in Bhavnagri (2012) for $N=3$ there are $k=3$ pointwise linearly independent vector fields on the lens space since $4=2^{2}(20+1), m=2$ and $k=3$ from $k=2 m-1$. It follows that this lens space admits free actions.

Above we saw that the Lens spaces $L(p, q)$ with $p$ and $q$ relatively prime and $q>2$ are not symmetric spaces. This meant that these spaces have no isometries (in the isotropy group) that are involutions. The lens spaces $L(3, q)$ with $q>2$ but relatively prime to 3 are therefore pairwise consistent, with respect to the structure of isometries.

Now since we know that those spin Hilbert spaces corresponding to free radicals are not pairwise consistent, and we also know that a lens space $L(3, q)$ with $q$ relatively prime to 3 is pairwise consistent, we suspect that the latter is a limit of the former. Consequently we construct the index for pairwise consistency of an atom or molecule as follows.

If $q$ is the total number of electrons we define the index to be q modulo 3 .

\section{EXAMPLES OF SUBSTANCES}

Water or $\mathrm{H}_{2} \mathrm{O}$ has 10 electrons and non-zero index. The table 1 lists the atmospheric gases, in decreasing order of concentration in the earths atmosphere. It can be seen that the only components of air with a zero index are Noble elements, except ozone which humans have little exposure to.

Next we consider Benzene $C_{6} H_{6}$, which is a known carcinogen. It has 42 electrons and a zero index. On the other hand Toluene $\mathrm{C}_{7} \mathrm{H}_{8}$ is not a carcinogen, has 50 electrons and a non-zero index 1 . The table 2 lists chemicals tested on rhesus monkeys from the carcinogenic potency database. 
Table 1. Atmospheric gases

\begin{tabular}{|c|c|c|c}
\hline & Molecular formula & Electrons & Index \\
\hline \hline Nitrogen & $\mathrm{N}_{2}$ & 14 & 2 \\
\hline Oxygen & $\mathrm{O}_{2}$ & 16 & 1 \\
\hline Argon & $\mathrm{Ar}$ & 18 & 0 \\
\hline Carbon Dioxide & $\mathrm{CO}_{2}$ & 22 & 1 \\
\hline Neon & $\mathrm{Ne}$ & 10 & 1 \\
\hline Helium & $\mathrm{He}$ & 2 & 2 \\
\hline Methane & $\mathrm{CH}_{4}$ & 10 & 1 \\
\hline Krypton & $\mathrm{Kr}$ & 36 & 0 \\
\hline Hydrogen & $\mathrm{H}_{2}$ & 2 & 2 \\
\hline Nitrous oxide & $\mathrm{N}_{2} \mathrm{O}$ & 22 & 1 \\
\hline Carbon Monoxide & $\mathrm{CO}$ & 14 & 2 \\
\hline Xenon & $\mathrm{Xe}_{3}$ & 54 & 0 \\
\hline Ozone & $\mathrm{O}_{3}$ & 24 & 0 \\
\hline Nitrogen Dioxide & $\mathrm{NO}_{2}$ & 23 & 2 \\
\hline Iodine & $\mathrm{I}_{2}$ & 106 & 1 \\
\hline Ammonia & $\mathrm{NH}_{3}$ & 10 & 1 \\
\hline
\end{tabular}

Table 2. Chemicals tested on rhesus monkeys from Carcinogenic potency database

\begin{tabular}{|c|c|c|c|}
\hline Chemical name & CAS & $T D_{50}$ & index \\
\hline 2-Acetylaminofluorene & 53-96-3 & & 1 \\
\hline 2,7-Acetylaminofluorene & $304-28-9$ & & 1 \\
\hline Adriamycin & $23214-92-8$ & & 1 \\
\hline Aflatoxin B1 & $1162-65-8$ & 0.0082 & 0 \\
\hline Arsenate, sodium & $7631-89-2$ & & 0 \\
\hline Azathioprine & $446-86-6$ & & 1 \\
\hline Cyclamate, sodium & $139-05-9$ & & 1 \\
\hline Cyclophosphamide & $50-18-0$ & & 1 \\
\hline DDT & $50-29-3$ & & 1 \\
\hline N,N-Dimethyl-4-aminoazobenzene & $60-11-7$ & & 0 \\
\hline IQ & $76180-96-6$ & & 2 \\
\hline Melphalan & $148-82-3$ & & 1 \\
\hline 3-Methyl-4-dimethylaminoazobenzene & $55-80-1$ & & 0 \\
\hline N-Methyl-N-nitro-N-nitrosoguanidine & $70-25-7$ & & 1 \\
\hline 3-Methylcholanthrene & $56-49-5$ & & 0 \\
\hline 2-Naphthylamine & $91-59-8$ & 5.74 & 1 \\
\hline N-Nitroso-N-methylurea & $684-93-5$ & 7.18 & 0 \\
\hline N-Nitrosodiethylamine & $55-18-5$ & 0.0536 & 2 \\
\hline N-Nitrosodimethylamine & $62-75-9$ & & 1 \\
\hline N-Nitrosodipropylamine & $621-64-7$ & 0.0121 & 0 \\
\hline N-Nitrosopiperidine & $100-75-4$ & 2.76 & 2 \\
\hline Procarbazine.HCl & $366-70-1$ & 4.21 & 0 \\
\hline Saccharin, sodium & $128-44-9$ & & 0 \\
\hline Sterigmatocystin & $10048-13-2$ & & 0 \\
\hline Urethane & $51-79-6$ & 65.2 & 0 \\
\hline
\end{tabular}


B. Bhavnagri, An index of carcenogenesis using pairwise consistency

The reader may notice that DDT has an index greater than 0 , but in fact it can be metabolized to DDE, which has an index of 0 . Thus one application of the index may be to identify which among a similar group of chemicals is the actual carcinogen.

\section{Conclusions}

We perform a $2 \times 2$ contingency analysis of the rhesus monkeys data from the carcinogenic potency database. Our null hypothesis is that the index is not significantly different between carcinogenic and non-carcinogenic chemicals. The observed and expected cell frequency tables follow.

\begin{tabular}{|c|c|c|c|}
\hline & Carcinogen & Not carcinogen & Total \\
\hline \hline Index $=0$ & 4 & 4 & 8 \\
\hline Index $>0$ & 1 & 10 & 11 \\
\hline Total & 5 & 14 & 19 \\
\hline & Carcinogen & Not carcinogen \\
\hline & \multicolumn{2}{|c}{5.894} \\
\hline Index =0 & 2.105 & 8.105
\end{tabular}

The computed value of $\chi^{2}$ is 3.997 which at $\alpha=0.05$ is greater than the tabulated $5 \%$ value of 3.84 . Therefore we reject the null hypothesis. The index is significantly different between carcinogenic and non-carcinogenic chemicals tested on rhesus monkeys.

\section{REFERENCES}

Bhavnagri, B. (2011). Representational consistency of group rings. Journal of Mathematics Research 3(3).

Bhavnagri, B. (2012). Representational consistency. Burzin Bhavnagri.

Carey, A. L., K. C. Hannabuss, V. Mathai, and P. McCann (1998). Quantum hall effect on the hyperbolic plane. Communications in Mathematical Physics 190(3), 629-673.

Halliwell, B. (1994). Free radicals, antioxidants, and human disease curiosity, cause, or consequence. The Lancet 344(8924), 721.

Penrose, R. (1994). On bell non-locality without probabilities: some curious geometry. In J. Ellis and A. Amati (Eds.), Quantum reflections in honour of J.S. Bell.

Zimba, J. and R. Penrose (1993). On bell non-locality without probabilities: more curious geometry. Stud. Hist. Phil. Sci. 24(5). Majorana 1932. 